DIGITALCOMMONS - @WAYNESTATE -
Clinical Research in Practice: The Journal of Team Hippocrates

2016

\title{
Induced hypothermia post cardiac resuscitation works in hospitalized patients but not during transport to hospital, a logical inconsistency of evidence
}

Joshua Frederick

Wayne State University, jfreder@med.wayne.edu

Follow this and additional works at: https://digitalcommons.wayne.edu/crp

Part of the Investigative Techniques Commons, Medical Education Commons, and the Translational Medical Research Commons

\section{Recommended Citation}

Frederick J. Induced hypothermia post cardiac resuscitation works in hospitalized patients but not during transport to hospital: A logical inconsistency of evidence. Clin. Res. Pract. Feb 5, 2016;2(1):eP1023. doi: 10.22237/crp/1454682640

This Critical Analysis is brought to you for free and open access by the Open Access Journals at DigitalCommons@WayneState. It has been accepted for inclusion in Clinical Research in Practice: The Journal of Team Hippocrates by an authorized editor of DigitalCommons@WayneState. 


\title{
Induced hypothermia post cardiac resuscitation works in hospitalized patients but not during transport to hospital: A logical inconsistency of evidence
}

\author{
JOSHUA FREDERICK, BA, Wayne State University, Detroit, MI, jfreder@med.wayne.edu
}

\begin{abstract}
A critical appraisal and clinical application of Kim F, Nichol G, Maynard C, et al. Effect of prehospital induction of mild hypothermia on survival and neurological status among adults with cardiac arrest. JAMA 2013;311(1):45-52.

doi:10.1001/jama.2013.282173
\end{abstract}

Keywords: hypothermia, cardiac arrest, prehospital

\section{Clinical Context}

A 54 year old African American male with history of end stage renal disease, human immunodeficiency virus, hypertension, coronary artery disease, chronic obstructive pulmonary disease, diastolic heart failure, hepatitis $\mathrm{C}$, and Kaposi sarcoma was initially admitted for uremia secondary to non-adherence with dialysis. During his stay, he was found to have a CD4 count of 16 and was persistently bacteremic with Vancomycin Resistant Enterococci from an unknown source for at least one month. His clinical condition continued to deteriorate as the source of his persistent bacteremia remained a mystery. As he was talking on the phone with a family member he suddenly went into respiratory distress with pulseless electrical activity. A code blue was initiated and ran for approximately 17 minutes before a pulse was identified and he was stabilized. Hypothermic protocol was then initiated by placing bags of ice on the patient's groin and armpits and the patient was sent to the intensive care unit (ICU) for further care.

Within 2 weeks of cardiac arrest, the patient had been extubated, was ambulating, following commands and his bacteremia had subsided. The patient was ready to be discharged. His rapid recovery encouraged me to learn more about hypothermia after cardiac arrest. I discovered it is common practice to perform therapeutic hypothermia post cardiac arrest for patients in the hospital, but I wondered if earlier intervention for community cardiac arrest might be more beneficial.

\section{Clinical Question}

Does inducing hypothermia for patients outside of the hospital improve their prognosis?

JOSHUA FREDERICK, BA, is a medical student at the Wayne State University School of Medicine. 
FREDERICK J. Critical appraisal and clinical application of Kim F, et al. Effect of prehospital induction of mild hypothermia on survival and neurological status among adults with cardiac arrest. JAMA 2013;311(1):45-52.
Clinical Research in Practice

The Journal of Team Hippocrates

VOL 2 ISS 1 / eP1023 / FEBRUARY 5, 2016

\section{Research Article}

Kim F, Nichol G, Maynard C, et al. Effect of prehospital induction of mild hypothermia on survival and neurological status among adults with cardiac arrest. JAMA 2013;311(1):45-52. doi:10.1001/jama.2013.282173

\section{Literature Review}

It has been shown that therapeutic hypothermia-lowering a patient's body temperature to between $32^{\circ}-34^{\circ} \mathrm{C}$ for 24 hours after being stabilized from cardiac arrest-improves survival and neurologic outcome ${ }^{2}$. In addition, Laver, et al. has evidence to suggest that there is no difference in outcomes between patients maintained at $33^{\circ} \mathrm{C}$ vs $36^{\circ} \mathrm{C}^{3}$. However, these studies focus primarily on inhospital treatment and there are only a few studies that investigate prehospital hypothermia. Aside from the article to be discussed, the only study of prehospital hypothermia for patients resuscitated from cardiac arrest without ventricular fibrillation (VF) had reduced power due to small sample size ${ }^{\underline{1}} \underline{4}$. This critical appraisal therefore looks to assess the quality of evidence of this larger study to determine if inducing therapeutic hypothermia prehospital provides clinically significant benefits.

\section{Critical Appraisal}

The studying being examined is a randomized, standard-of-care-controlled study using intention to treat analysis. It demonstrates Level $1 \mathrm{~b}$ evidence according to the Oxford Centre for Evidence Based Medicine. Of 2,377 eligible patients, 1,359 patients served by Seattle and King County, Washington, emergency medical services were stratified according to their first recorded rhythm (583 with VF and 776 without VF). They were then randomly assigned to the intervention group, using intravenous (IV) infusion up to $2 \mathrm{~L}$ of $4^{\circ} \mathrm{C}$ normal saline (NS) through a peripheral vein, or standard of care after spontaneous return to circulation following cardiac arrest. Of the 2,377 patients who were eligible, 497 were missed, 211 were deemed too unstable by the paramedics, 5 were withdrawn from the study because they were incarcerated and 305 were not enrolled due to other reasons such as equipment failure or hospital arrival before randomization. Thus half the patients eligible for enrollment were not included in the study. Protocol violations of this magnitude introduce selection bias large enough to affect the intention to treat analysis and study outcome. There was no reported description of "deemed too unstable by the paramedics." The prehospital cooling decreased core body temperature prehospital by $1.2^{\circ} \mathrm{C}$ and $1.3^{\circ} \mathrm{C}$ for patients with VF and without VF, respectively, and was compared to the standard of care. If recurrent arrest occurred during transport, standard resuscitation was initiated and the IV infusion was stopped until circulation returned. After arriving at the hospital, all VF patients and 1 hospital receiving patients without VF used cooling protocols for up to 24 hours. $T$-test for normal variables, Wilcoxon rank sum test for non-normally distributed variables and Chi-squared statistic for categorical variables were used to analyze the data. It was concluded that although prehospital induction of hypothermia led to significantly faster cooling times once in the hospital, there was no statistical difference in survival or neurologic outcomes between the intervention and control.

Although this study had increased power compared to previous studies, there were some shortfalls that should be addressed. First, while they did increase their power compared to previous studies, their assumption of differences in survival rates with the intervention and control ( $65 \%$ and $50 \%$, respectively, for patients with VF) was inaccurate, with both survival rates being closer to 63-64\%. However, by increasing power, the authors could only have found clinically meaningless differences. There was also the possibility of bias from the healthcare workers who knew the treatment of each patient. Confounding was another shortfall that could have influenced this study. For instance, those treated with prehospital NS also took a statistically significant longer time to arrive at the hospital (51 min vs. $49 \mathrm{~min}$ ) delaying crucial, time-dependent care. This increased time to the hospital could account for the statistically significant lower $\mathrm{pH}$ (indicating worse prognosis) and more episodes of re-arrest. In addition, the adverse effects of the IV infusion could have negated the positive effects from early therapeutic hypothermia. Those in the intervention group had increased transient pulmonary edema that could have been a result of fluid overload. Therefore, this study failed to show benefit and demonstrated increased complications with prehospital induction of hypothermia using normal saline. However, many of the complications may have been related to the use of NS for induction and it is not clear whether other methods of cooling may produce more favorable results. 


\section{Clinical Application}

The method of inducing hypothermia in my patient differed in comparison to those enrolled in the study reviewed. Intuitively, the earlier the treatment, the better the expected outcome, yet this was not demonstrated with this critical appraisal. Until we understand the role of hypothermia in these patients better, NS infusions to initiate therapeutic hypothermia during transport to the hospital should not be used due to the lack of proven benefit in improving survival and neurologic outcome and possibly causing unnecessary harm to the patient. It is, however, logically inconsistent that induced hypothermia is beneficial in the hospital but not during transport to the hospital. Due to the time sensitive nature of treating cardiac arrest, more studies should be done to determine if other modalities of cooling can be used prehospital to improve a patient's prognosis.

Three learning points:

1.) When interpreting a study it is important to look for areas of confounding that can skew results.

2.) Normal saline should not be used prehospital to induce hypothermia.

3.) More research is needed to understand the logical inconsistency between outcomes of prehospital induction of hypothermia compared to hospital induction of hypothermia for post cardiac arrest.

\section{References}

1. Kim F, Nichol G, Maynard C, et al. Effect of prehospital induction of mild hypothermia on survival and neurological status among adults with cardiac arrest. JAMA 2013;311(1):45-52. doi:10.1001/jama.2013.282173

2. Arrich J, Holzer M, Havel C, Müllner M, Herkner H. Hypothermia for neuroprotection in adults after cardiopulmonary resuscitation. Cochrane Database Syst Rev 2012;9:CD004128. doi:10.1002/14651858.CD004128.pub3

3. Nielsen $\mathrm{N}$, Wetterslev J, Cronberg T, et al. Targeted temperature management at $33^{\circ} \mathrm{C}$ versus $36^{\circ} \mathrm{C}$ after cardiac arrest. $N$ Engl J Med. 2013;369(23):2197. doi:10.1056/NEJMoa1310519

4. Bernard SA, Smith K, Cameron P, et al. Induction of prehospital therapeutic hypothermia after resuscitation from nonventricular fibrillation cardiac arrest. Crit Care Med. 2012;40(3):747-753. doi:10.1097/CCM.0b013e3182377038. 